\title{
À l'épreuve des pères : la phratrie congolaise
}

\section{Céline Gahungu}

\section{(2) OpenEdition}

Journals

Édition électronique

URL : https://journals.openedition.org/genesis/5707

DOI : 10.4000/genesis.5707

ISSN : 2268-1590

\section{Éditeur :}

Presses universitaires de Paris Sorbonne (PUPS), Société internationale de génétique artistique littéraire et scientifique (SIGALES)

\section{Édition imprimée}

Date de publication : 15 décembre 2020

Pagination : 127-140

ISBN : 979-10-231-0704-3

ISSN : 1167-5101

\section{Référence électronique}

Céline Gahungu, «À l'épreuve des pères : la phratrie congolaise », Genesis [En ligne], 51 | 2020, mis en ligne le 20 décembre 2021, consulté le 07 février 2022. URL : http://journals.openedition.org/genesis/ 5707 ; DOl : https://doi.org/10.4000/genesis.5707 


\title{
À l'épreuve des pères : la phratrie congolaise
}

\author{
Céline Gahungu
}

$\mathrm{A}^{\mathrm{u}}$ u début des années soixante, le Congo traverse une période instable marquée par des procès et des assassinats politiques, des coups d'État et des tensions ethniques avivées. Les soubresauts qui agitent le corps social atteignent un pic en 1968 : un militaire, Marien Ngouabi, renverse le président Alphonse Massamba-Débat, s'empare du pouvoir et fonde le Parti congolais du travail. Inspiré du modèle soviétique, le Parti encadre, peu à peu, la vie politique, économique et culturelle de la République populaire du Congo; les rumeurs et le contrôle croissant exercé sur la presse écrite et la radiodiffusion alimentent une atmosphère pesante à laquelle nul ne peut échapper.

Ces difficultés n'empêchent pas certains Congolais de se vouloir écrivains ; à partir du milieu des années soixante, ce désir prend, chez quelques-uns, une dimension collective. Henri Lopes, Jean-Baptiste Tati Loutard et Maxime Ndébéka se rencontrent et commentent leurs écrits pour l'essentiel inédits. Sous la poussée des événements politiques, ce premier cercle s'élargit et commence une mue dont l'un des acteurs principaux est Sylvain Bemba : après avoir connu l'amertume de la proscription en raison d'un pamphlet qu'on l'accuse d'avoir diffusé clandestinement, Autocritique du M22, il décide d'aider de jeunes écrivains ${ }^{1}$. Romanciers, poètes et dramaturges créent un territoire littéraire commun où, à l'abri des clivages idéologiques, manuscrits et idées s'échangent librement : la phratrie. C'est à Sylvain Bemba, son chef de chœur, que l'on doit ce nom :

Dans son étude sur l'origine de la famille, Engels s'est penché sur un groupe particulier existant chez les tribus indiennes, et que Morgan «traduisant fidèlement le nom indien, appelle phratrie (fraternité) d'après son pendant grec.» C'est ce même nom que nous avons décidé de retenir pour caractériser les liens peu banals qui unissent la plupart des écrivains congolais. Bien qu'habitant des lieux séparés, ces derniers se retrouvent là «où souffle l'esprit», en Congolie, région imaginaire réservée à la fiction, à la création, des œuvres de beauté, véritable espace de convivialité et principauté de l'esprit...2
Des années soixante jusqu'à la décomposition de la République populaire une vingtaine d'années plus tard, la phratrie se constitue selon des logiques proches d'autres groupes littéraires tout en se dotant de ressorts singuliers. Elle n'est guère la gardienne de codes esthétiques et n'est pas régulée par une sociabilité de salon. Ni cénacle, ni école, elle est le lieu d'un être-ensemble informel tissé au fil des rencontres dans les bars brazzavillois, chez les écrivains et un couple de coopérants français, tous deux professeurs de lettres au sein de l'université Marien-Ngouabi, Arlette Chemain-Degrange et Roger Chemain. L'hétérogénéité sociale garantit la survie de la phratrie. Dans un jeu littéraire et politique complexe, d'éminents ministres évitent dangers et chausse-trappes à des jeunes gens pressés de publier. Un imaginaire familial façonne cette petite société : fondée sur la transmission filiale d'un savoir-faire littéraire, elle est peuplée de fils et de cadets qui cheminent avec confiance, protégés par l'ombre tutélaire de leurs pères, parrains et aînés.

Dans ce laboratoire créatif caractérisé par des échanges permanents, quelles sont les formes et les spécificités de l'exogenèse? Les craintes suscitées par les répressions exercées au sein de la République populaire, les relations denses nouées entre les écrivains et la mise en œuvre de démarches créatrices collaboratives semblent constituer la phratrie en objet tout indiqué pour mesurer la puissance heuristique d'une exogenèse analysée dans son extension la plus large. Le matériel exogénétique, en effet, concerne aussi bien des éléments intertextuels que des documents de recherche et des écrits où sont rapportés, parfois dans l'urgence du hic et nunc, les événements qui agitent le corps social.

1. Sur cette période, on peut consulter l'autobiographie d'Henri Lopes, Il est déjà demain (Paris, Lattès, 2018).

2. Sylvain Bemba, «La phratrie des écrivains congolais», Notre Librairie, no 92-93, «Littérature congolaise», mars-mai 1988, p. 13. 
À cette projection, il faut opposer les réalités de la constitution et de la conservation des archives littéraires congolaises. Pour des raisons liées aux guerres civiles, aux exils, au spectre des violences, et dans certains cas, au désintérêt pour l'archivage, les fonds actuels sont lacunaires. À l'exception d'un inventaire publié par Nicolas Martin-Granel et Greta Rodriguez-Antoniotti dans la revue Études littéraires africaines, il ne reste aucune trace des bibliothèques de la phratrie ${ }^{3}$. La maison de Sylvain Bemba, où se trouvaient une importante bibliothèque, des brouillons et des correspondances, a été pillée et détruite en 1998. Les manuscrits de Maxime Ndébéka ont en partie disparu et les archives de Tchicaya U Tam'si et de Jean-Baptiste Tati Loutard sont, pour la plupart d'entre elles, inaccessibles. Seuls Sony Labou Tansi et Henri Lopes, à notre connaissance, ont pensé le devenir d'une partie de leurs manuscrits et tapuscrits. Les archives d'éditeurs - au premier chef, celles du Seuil et de la maison d'édition CLÉ, située à Yaoundé - abritent elles aussi de précieux gisements qui demandent encore à être inventoriés.

Les fonds qu'il est possible de consulter présentent une caractéristique : les documents exogénétiques qui prennent la forme de repérages, de correspondances à visée informative ou de notes élaborées à partir d'éléments lus, vus, entendus ou rapportés n'ont, jusqu'à présent, jamais été observés ni mentionnés. Ces fragments pré-rédactionnels ont-ils échappé à la vigilance des chercheurs? Les écrivains les ont-ils détruits ou perdus? Devant l'ampleur du phénomène, l'hypothèse qu'ils n'aient jamais existé est la plus probable.

Est-ce à dire que s'intéresser à la phratrie sous l'angle de l'exogenèse est voué à l'échec ? Bien au contraire. Si les échanges réguliers des écrivains ont favorisé des réseaux intertextuels dont il reste à étudier les usages et les effets au fil des manuscrits et tapuscrits qui nous sont parvenus, l'existence d'une autre forme d'exogenèse, problématique puisqu'elle aurait échappé à l'écrit, est à considérer : l'enjeu est de taille, sur le double plan de l'histoire littéraire et de la théorie. Dans quelle mesure une exogenèse polymorphe, circulant d'un manuscrit à l'autre, parfois constituée de séquences manquantes, est-elle une voie pour étudier la phratrie au-delà du fantasme collectif sur lequel elle s'est fondée?

\section{L'ombre des pères}

\section{Fable : l'écrivain et le miroir magique}

«77 sanglots pour nègre congo 4 », nouvelle que Sylvain Bemba compose peu de temps avant sa mort, est à bien des égards une réflexion sur l'exogenèse. Dans ce récit métatextuel qui condense les interrogations partagées avec ses amis, transparaissent des dynamiques créatrices devenues, au fil du temps, communes à l'ensemble de la phratrie.

Le texte est structuré par un dispositif narratif complexe : il fait alterner les voix du peintre Godden, sur le point de sombrer dans la folie, et d'un ami qui observe avec inquiétude les signes d'un égarement inéluctable.

Lorsque l'intrigue commence, Godden travaille à son tableau le plus ambitieux, une œuvre consacrée à un épisode sanglant de la vie politique congolaise et longtemps passé sous silence : le meurtre, en 1977, de Marien Ngouabi, suivi d'une série d'assassinats politiques. Godden souhaite convertir le traumatisme national en matériau artistique à l'ombre d'un modèle : Carpaccio. Une nuit, le peintre lui est apparu en songe et lui a enjoint, pour créer sa toile, de s'inspirer de ses Lamentations sur le Christ mort. Le processus créatif qui s'ensuit semble être un échec. Malgré les conseils de Carpaccio, Godden se refuse à noter sa vision, délaisse les événements politiques qu'il devait traiter et ne fait rien de la toile du maître qu'il parvient à contempler grâce à un miroir magique, un «talisman-songe » dont les enchantements abolissent les distances et offrent à ses regards les objets qu'il convoite.

Lors du vernissage, un scandale éclate. Le Vendredides-douleurs est une toile vide qui suscite ce commentaire chez son auteur : «Ce travail existe puisque ma tête l'a fait. Quand le ventre de la femme porte un enfant, tu veux dire que l'enfant n'existe pas puisque tu le vois pas?» Désavoué et incompris, Godden se retire dans une secte messianique où, au cours de mystérieuses séances thérapeutiques,

3. Nicolas Martin-Granel, Greta Rodriguez-Antoniotti (dir.), «Inventaire de la bibliothèque de Sony Labou Tansi », Études littéraires africaines, no 15, «Approche génétique des écrits littéraires africains. Le cas du Congo », 2003, p. 39-46 (en ligne sur erudit.org).

4. Sylvain Bemba, «77 sanglots pour nègre congo », Africultures, 30 novembre 1998. Jean-Michel Devésa, "Genèse d'une nouvelle de Sylvain Bemba», loc. cit. 
son corps, recouvert chaque jour de nouveaux signes tracés au kaolin, devient peu à peu un objet artistique total, tout à la fois peinture, texte et sculpture.

Dans cette fable mélancolique traversée par des échos au Chef d'œuvre inconnu, Sylvain Bemba met en scène une création avortée : si l'élaboration d'un projet artistique et sa réception forment la trame de la nouvelle, le travail de l'artiste sur sa toile en est le point aveugle. L'exogenèse tient une place importante dans l'esprit du peintre, inquiet de la démarche à concevoir. Jusqu' où doit-elle être informée par un modèle qui, loin de stimuler sa créativité, risque de la paralyser? Godden le pressent : «Faut jamais voir un truc pareil quand tu es apprenti, sinon tu préfères rester toute ta vie chômeur de la peinture.» Théorisée par Harold Bloom et, à la suite de ses travaux, par Jean-Louis Cornille et Anthony Mangeon 5 , l' «angoisse de l'influence » est si puissante que le personnage supprime de la manière la plus radicale une confrontation artistique vouée à la frustration et au naufrage. En refusant l'assimilation endogénétique des Lamentations sur le Christ mort, le peintre est le seul à jouir de son rêve esthétique. De même, les événements politiques au centre du Vendredi-des-douleurs ont donné lieu à une recherche sur la figuration picturale de personnages historiques, mais rien ne demeure de ce travail préalable, sinon un titre et les souvenirs des narrateurs.

Via Godden transformé en œuvre vivante à la fin de la nouvelle, Sylvain Bemba suggère que si les convulsions du Congo révolutionnaire et les manuscrits lus au gré des rencontres ont été l'aiguillon de projets artistiques, les écrivains de la phratrie les ont intégrés selon des modalités qui en rendent les traces parfois indécelables. Tantôt pleinement intériorisée, tantôt à l'œuvre dans des fragments disparus, tantôt perceptible au fil des manuscrits conservés, une exogenèse complexe travaille sourdement les processus créatifs déployés par les écrivains. Sur le modèle du miroir magique de Godden et selon des reflets changeants, les écrits de la phratrie réfléchissent un environnement, un milieu social et d'autres textes.

Caractérisée par une lacune documentaire puisque la genèse de ses récits composés au Congo est pour l'essentiel perdue, la première partie de la trajectoire littéraire d'Henri Lopes est à mettre en perspective avec la réflexion de Sylvain Bemba. De 1969 à 1981, Henri Lopes, tour à tour ministre de l'Éducation nationale, ministre des Affaires étrangères, Premier ministre, puis ministre des Finances, s'efforce de transformer sa position, située à la croisée des chemins, en une dynamique littéraire : imaginer un univers dont la politique et le désir de devenir écrivain seraient la pâture. Menés entre le printemps 2018 et l'automne 2019, nos entretiens relatifs à ses premiers écrits 6 ne peuvent remplacer leurs archives disparues, toutefois les souvenirs de l'écrivain indiquent l'existence, avant toute rédaction, d'une phase de création intériorisée. À l'origine de leur gestation, ses premiers projets sont étroitement liés à la vie politique et sociale congolaise, et plus largement francoafricaine. Il observe l'exercice du pouvoir, recueille les confidences d'amis également en poste et «note ces scènes, dans [s]on esprit, afin de les transformer en romans ${ }^{7}$ ». C'est ainsi que les confidences d'Enoch Lakoué 8 sur les habitudes fantasques de Bokassa ont nourri les campagnes d'écriture du Pleurer-Rire. Cette exogenèse intériorisée est le lot d'autres écrivains. Sous l'étroite dépendance d'une actualité brûlante, parfois menacée, la genèse des œuvres s'inscrit dans un tempo rapide qui incite à une double opération, quasi immédiate, de fictionnalisation/textualisation.

Les manuscrits de Sony Labou Tansi et sa correspondance avec Françoise Ligier, à la tête des programmes africains de l'Office de coopération radiophonique, et José Pivin, producteur d'émissions radiophoniques pour l'ORTF, laissent transparaître les ressorts d'une création dans la gueule du loup. Une lettre datée du 15 septembre 1974 porte sur le nouveau projet de l'écrivain. Fasciné

5. Harold Bloom, L'Angoisse de l'influence, Paris, Éditions Aux Forges de Vulcain, 2013. Jean-Louis Cornille, Plagiat et Créativité II : Douze enquêtes sur l'auteur et son double, Amsterdam, Rodopi, coll. «Faux Titre», 2011. Anthony Mangeon, Crimes d'auteur : de l'influence, du plagiat et de l'assassinat en littérature, Paris, Hermann, coll. «Fictions pensantes », 2016.

6. Tribaliques, La Nouvelle Romance, Sans Tam-Tam et Le Pleurer-Rire. 7. Entretien consacré au Pleurer-Rire, 4 juin 2019. Dans Il est déjà demain, Henri Lopes décrit ses débuts de Premier ministre en ces termes : «Durant cette période où j'étais un acteur important de l'histoire du pays, je n'ai pas tenu de journal. Je me suis contenté de prendre des notes sur des situations, des individus, des atmosphères, des détails, en vue des romans que je ne désespérais pas d'écrire un jour. » Mise en perspective avec l'ensemble de ses écrits, cette remarque suggère à quel point la question de l'exogenèse est délicate. Henri Lopes, Il est déjà demain, op. cit., p. 367.

8. Enoch Lakoué occupe des fonctions ministérielles dans le cadre du régime instauré par Bokassa. 
par Alphonse Massamba-Débat, le président déchu qu'il vient de rencontrer à Boko, Sony Labou Tansi ambitionne un écrit destiné à lui rendre hommage, Massamba-Débat, l'homme tout court ${ }^{9}$. Ni sa forme ni son contenu ne sont décrits, mais sa rédaction aurait sans doute été conçue sur le modèle d'un essai inabouti évoqué à la même période, La Délinquance idéologique, manière de discussion amicale avec José Pivin et Françoise Ligier. Rien ne subsiste des entretiens avec Massamba-Débat et, quelques semaines après le 15 septembre 1974, la correspondance annonce que l'hagiographie rêvée se métamorphose en matériau romanesque - il s'agit du chantier intitulé La Raison, le pouvoir et le béret. Qu' apprend-on à la lecture des cahiers 10 qui en ont résulté ? Que, dès les processus initiaux, les poussées de la création sont directement reportées sur les pages des manuscrits et empruntent les détours de la fiction : Massamba-Débat devient l'oncle Okemba; les conversations de l'automne 1974 se transforment en échanges filiaux qui, cahiers après cahiers, s'amenuisent pour laisser place à une intrigue de plus en plus complexe et ramifiée.

9. Nicolas Martin-Granel, Greta Rodriguez-Antoniotti (éd.), L'Atelier de Sony Labou Tansi, vol. I, Correspondance (1973-1983), Paris, Éditions Revue noire, coll. «Soleil», 2005, p. 152.

10. Conservés par la Bibliothèque francophone multimédia de Limoges, les manuscrits de Sony Labou Tansi sont, pour la plupart d'entre eux, des cahiers de petit format qui ne sont pas datés.

\section{Extrait de la correspondance de Sony Labou Tansi et Françoise Ligier*}

6 septembre 1974

Je suis à Kindamba où je prends mes affaires. Comme tu peux t'en rendre compte par cette nouvelle adresse que je te donne, on me mute au CEG Boko. À moins de $150 \mathrm{~km}$ de Brazzaville. La route est goudronnée jusque-là, puisque c'est le coin de l'ancien président de la République, qui aujourd'hui y fait pousser ses carottes et ses queues de persil. Il s'appelait Alphonse MassambaDébat. [...] Le CEG Boko est l'un des plus anciens du Congo et le plus grand hors de Brazzaville. (Près de 1500 élèves.) Mais cela je m'en fous. Une troupe théâtrale pourrait bien marcher là-dedans. Mais c'est une zone dangereuse à cause de la présence de l'Ancien président du Congo. Les bérets la surveillent sauvagement et la délinquance idéologique actuelle ne pardonne pas. (p. 151)

15 septembre 1974

C'est un homme, pas un béret lui. Et j'ai envie de lui faire la cour. Pendant les quatre jours que j'ai été laisser mes affaires là-bas, nous avons bavardé. J'ai trouvé le titre du bouquin : Massamba-Débat, L'Homme tout Court. On verra bien. Il faut te dire que ce n'est pas bien d'écrire sur un homme de son envergure mais je commence à me rendre compte que la vie, pour qu'elle ne vous embête pas, il faut l'embêter, s'en servir. (p. 152)

2 octobre 1974

Le bouquin avec le Pt, je ne suis pas bête à prendre des risques inutiles. Et puis, on dirait que je crois aux mots de de Gaulle qui disaient qu'on n'arrête pas Voltaire (même si je ne suis pas encore Voltaire et même si cela dépend des pays). Par deux fois j'ai causé avec le mec et j'en tire ma part. Ce qui est sûr c'est qu'on ne me prendra jamais dans un filet à putschistes. Je suis littéralement contre les coups de Régime. Il faut seulement informer les gens et tailler l'opinion. Voltaire et ses copains ont fait du mal au despotisme, ils n'étaient pas toujours en position de Dieu. Et puis le livre je l'écris sous forme romancée avec transposition de cadre. (p. 156)

3 octobre 1974

Je laisse tomber le bouquin sur le Pt. J'ai d'autres thèmes aussi stratégiques que celui-là. Je le reprends dès que tout marche. Au fond, on peut bien se foutre des instants politiques et créer en profondeur jusqu'à ce que ça germe. (p. 160)

29 septembre 1976

Boko. Effectivement c'est tout à fait idiot d'en partir. En plus de Nouany et Dihoulou et tant d'autres j'y avais le vieux Pt. Il m'instruisait. Tu verras que Le Trou que je t'envoie parle un peu de lui. Ainsi que La Raison et le béret. (p. 189)

* «Correspondance avec Françoise Ligier», dans N. Martin-Granel, G. Rodriguez-Antoniotti (éd.), L’Atelier de Sony Labou Tansi, vol. I, Correspondance, op. cit., p. 51-189. 


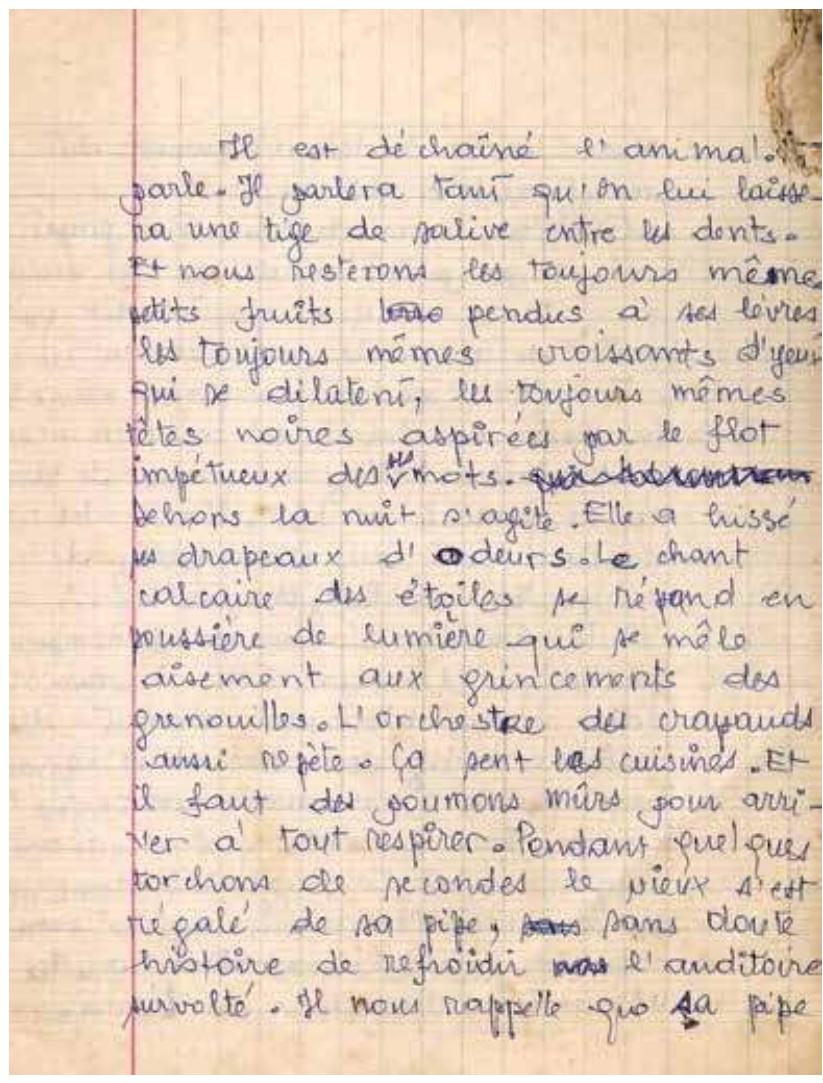

Fig. 1 à 3 : Sony Labou Tansi, cahier sans titre et sans date, brouillons de La Raison, le pouvoir et le béret.

(C) Bibliothèque francophone multimédia de Limoges, RES. PF SLT 33

La comparaison des lettres adressées à Françoise Ligier avec l'un des premiers cahiers qui se rattache à l'écriture de La Raison, le pouvoir et le béret restitue l'articulation entre une exogenèse intériorisée et une textualisation polarisée par une fictionnalisation immédiate. En partie fondé sur des entretiens, l'hommage à Alphonse Massamba-Débat semble abandonné en octobre 1974. L'entreprise, selon l'écrivain, serait bien trop dangereuse. À la lumière de la correspondance échangée avec Françoise Ligier entre 1974 et 1976, la situation est toutefois plus complexe qu'il n'y paraît. Quel sort réserver à ce matériau exogénétique que Sony Labou Tansi n'a pas pris en notes, mais conservé dans sa mémoire? Une lettre rédigée le 2 octobre 1974 constitue une réponse : le dévier vers un autre écrit cette fois-ci conçu «sous forme romancée avec transposition de cadre».

Ces pages du manuscrit de La Raison, le pouvoir et le béret dévoilent comment s'opère ce travail de fictionnalisation. Si la relation quasi filiale entre le narrateur, Ystèr, et son mentor structure le roman, et si la trajectoire d'Okemba est en tout point identique à celle d'Alphonse Massamba-Débat, la dynamique endogénétique de la fiction l'emporte sur toute autre considération. Autofiction fantasmatique dans laquelle l'écrivain projette une revanche sociale, politique et existentielle, La Raison, le pouvoir et le béret a pour héros, non pas Okemba, mais le génial et charismatique Ystèr, poète, essayiste et flamboyant opposant politique à un régime autocratique.
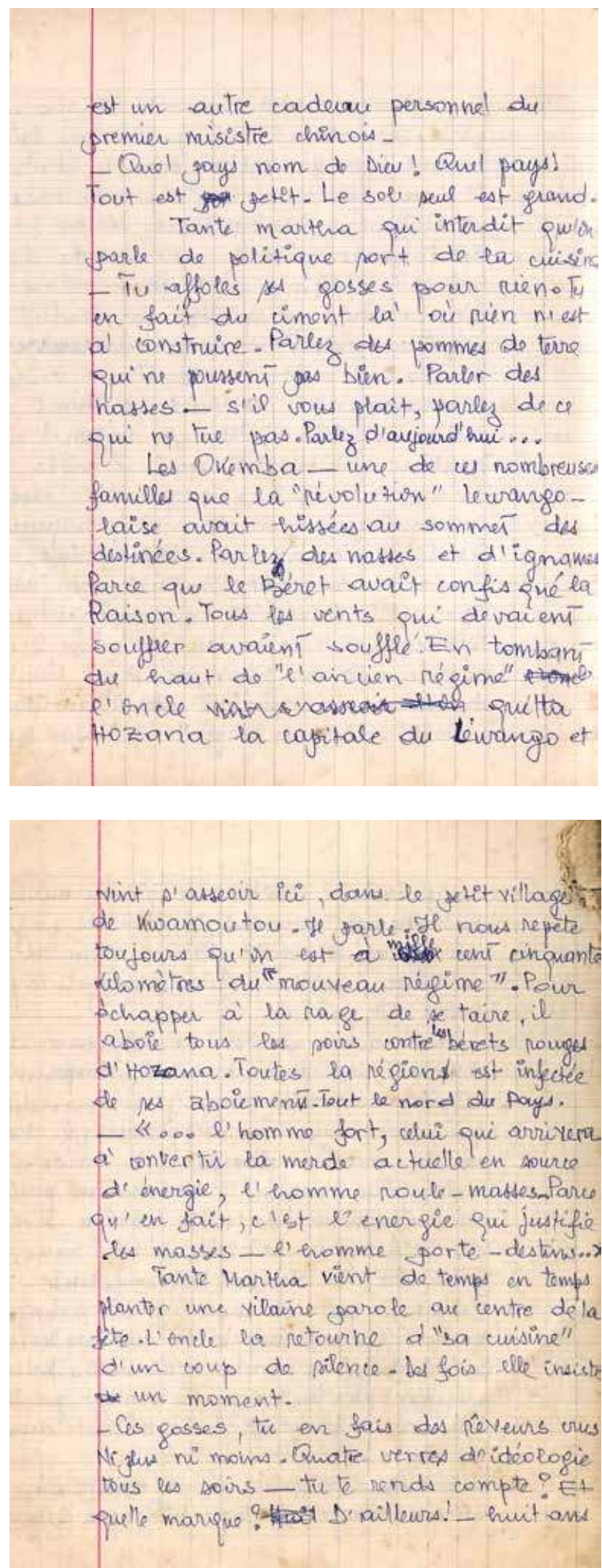
Une autre raison explique ce travail intériorisé : le désir de promouvoir la littérature congolaise francophone à la suite de l'Indépendance et dans le contexte d'un discours critique à l'encontre de la Négritude - Jean-Baptiste Tati Loutard est d'ailleurs l'auteur d'articles et d'anthologies à valeur de manifestes. L'épigraphe d'un roman inédit de Sony Labou Tansi, La Planète des signes, pourrait figurer au seuil des œuvres de ses pairs : "Quand j'écris un roman, c'est tout qui ne cesse de me dire : t'es pas un historien, mais un artiste11. ${ }^{11}$ Pour la plupart hommes politiques, journalistes, enseignants ou universitaires, les auteurs de la phratrie ont ressenti une angoisse identique - se trouver pris au piège de la référentialité et de la transparence, être des chroniqueurs et non des écrivains mus par des ambitions littéraires.

\section{Circulations}

Au fil des manuscrits de Sony Labou Tansi, se manifeste l'une des spécificités de l'exogenèse pratiquée dans la phratrie : l'existence d'une dynamique collaborative dont les modalités sont diverses, d'un écrit à l'autre. Les archives et les témoignages sont unanimes - Sylvain Bemba est l'âme de la phratrie et c'est vers lui que les écrivains se tournent afin qu'il lise leurs manuscrits et dispense ses conseils avisés. Son engagement dans la vie culturelle congolaise, son érudition, ses publications et sa réflexion aiguë sur les rapports entre les écrivains et le pouvoir en font un interlocuteur privilégié.

Dans son article «L'auteur et ses "nègres" : le cas de Sony Labou Tansi », Nicolas Martin-Granel s'intéresse au rôle de lecteur-correcteur joué par Sylvain Bemba auprès de son cadet à partir de la fin des années soixante-dix. Dans un manuscrit intitulé La Natte, se trouvent des notes allographes qui dévoilent le travail de Sylvain Bemba; l'une d'entre elles suscite l'intérêt du chercheur :

[D]ans cette annotation mise en évidence dans la marge du haut, Sylvain prend du recul pour commenter son travail, en marquer les limites, en s'adressant directement à Sony : «Partout, j’ai corrigé : emmerdant. Mais il se peut que tu aies voulu mettre merdant. Je le laisse partout, car tu l'as fabriqué12.»

La volte-face de Sylvain Bemba, qui se ravise puis salue l'invention de son ami, et le maintien de l'adjectif «merdant » suggèrent que Sony Labou Tansi ne s'en laisse pas conter. Le scepticisme un temps adopté par son aîné n'a guère donné le branle à une nouvelle séquence endogénétique au cours de laquelle le néologisme aurait été supprimé. Sony Labou Tansi, il est vrai, ne prend pas en compte le conseil stylistique de Sylvain Bemba. Il est vrai aussi que La Natte, à notre connaissance, est le seul manuscrit sur lequel apparaissent des commentaires de Sylvain Bemba. Pourtant, il importe de prendre en compte un indice souligné par Nicolas Martin-Granel, examiné ici sous un angle exogénétique. La volonté de recueillir, dans ses cahiers annotés et des correspondances, les impressions de lecture d'amis et la fascination exercée par l'écriture collective, depuis les lettres échangées avec José Pivin jusqu' aux créations dramaturgiques du Rocado Zulu Théâtre, dévoilent l'existence d'une exogenèse dialogique et collaborative.

Les dédicaces, dans lesquelles se laissent parfois percevoir les échos d'un imaginaire filial, en sont un indice. Reportée sur l'un des manuscrits de La Vie et demie, mais aussi dans le roman tel qu'il a été publié en 1979, une dédicace est adressée par l'écrivain à ses pères, Sylvain Bemba et Henri Lopes (fig. 4).

Dans les dispositifs imaginés par les écrivains, les dédicaces sont un espace de premier plan : lettres ouvertes, elles donnent parfois des pistes pour recomposer la genèse des écrits. Si l'importance du compagnonnage littéraire de Sylvain Bemba et Sony Labou Tansi est indéniable, ce sont les relations de ce dernier avec Henri Lopes qui vont jouer un rôle décisif dans les mutations de sa poétique romanesque. La pleine expression de ce qu'on pourrait appeler la maturité du romancier apparaît moins dans l'écriture de La Vie et demie que dans un projet évoqué en 1978 et intitulé au gré de ses métamorphoses Le Mort te dit adieu toi qui restes vivant, Machin la Hernie et L'État Honteux ${ }^{13}$; une dédicace inédite conservée à l'IMEC exprime toute

11. Sony Labou Tansi, La Planète des signes, Bibliothèque francophone multimédia de Limoges, RES. PF SLT 40, p. 16.

12. Nicolas Martin-Granel, «L'auteur et ses "nègres": le cas de Sony Labou Tansi », dans A. Begenat-Neuschäfer, C. Mazauric (éd.), La Question de l'auteur en littératures africaines, acte du 14e congrès de 1'APELA, 22-24 septembre 2011, Francfort-sur-Main, Peter Lang Edition, coll. «Sprachen Literaturen Kulturen. Reihe B, Sammelwerke », 2015, p. 45.

13. Sony Labou Tansi, Machin la Hernie, dans N. Martin-Granel, G. RodriguezAntoniotti (dir.), L'Atelier de Sony Labou Tansi, op. cit., vol. III. 

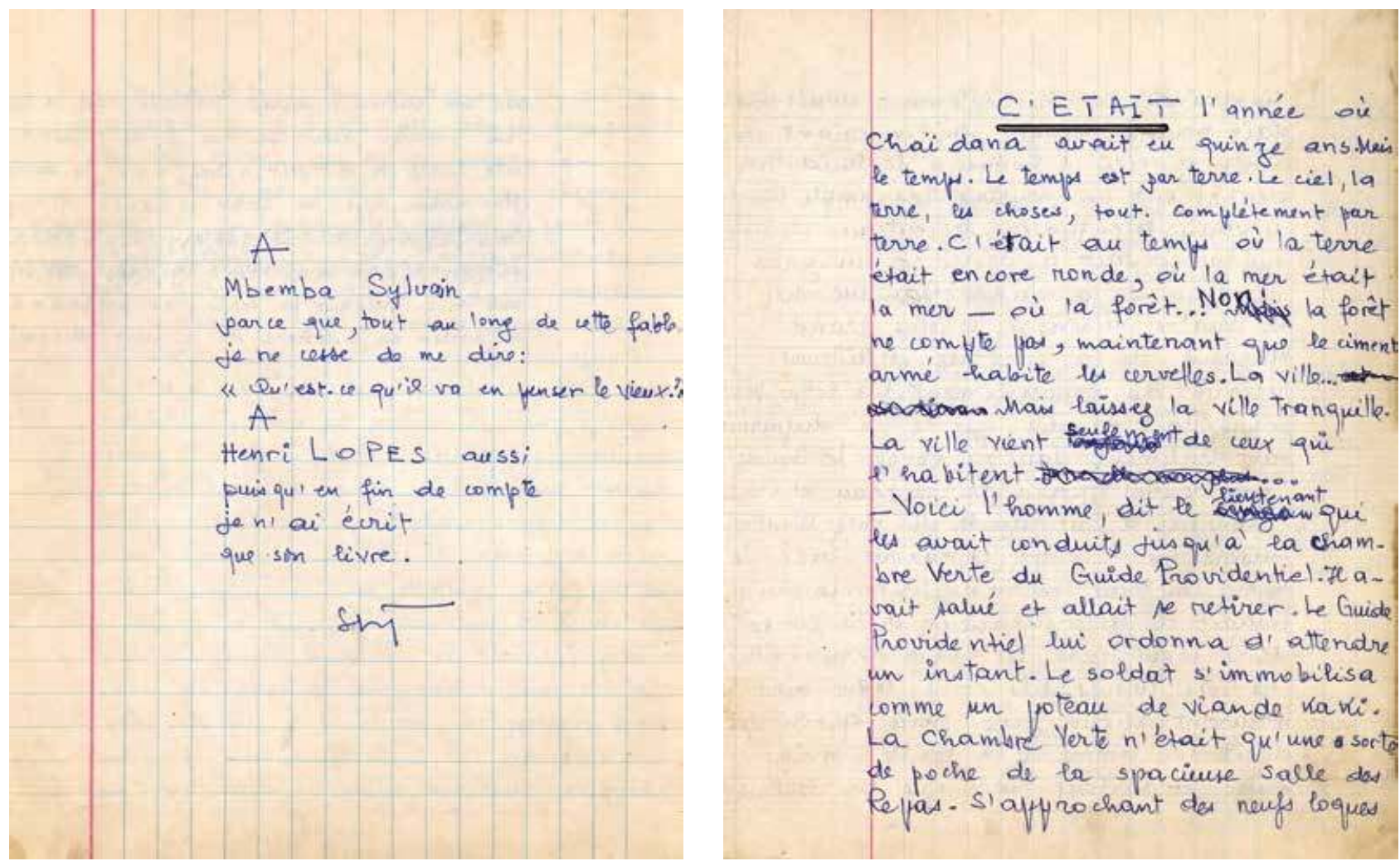

Fig. 4 : Sony Labou Tansi, manuscrit de La Vie et demie, p. 5-6.

L'écriture de La Vie et demie commence en 1977, période au cours de laquelle l'écrivain fréquente Sylvain Bemba et Henri Lopes. C'est précisément en 1977 que ce dernier invite Sony Labou Tansi à lire le manuscrit du roman qu'il est en train de composer, Le Pleurer-Rire. Selon la dédicace, La Vie et demie et Le Pleurer-Rire sont des récits jumeaux, puisque l'un serait la réécriture de l'autre.

(c) Bibliothèque francophone multimédia de Limoges, RES. PF SLT 50

l'affection de Sony Labou Tansi pour son ami : « À mon frère Henri Lopes cet instinct d'amour et de peur qui rit ${ }^{14}$.»

Entre 1975 et 1977, Henri Lopes s’éloigne temporairement de la vie publique et profite de cette période de vacance pour se consacrer davantage à l'écriture. Au moment de revenir aux affaires, en 1977, un roman polarise son énergie créatrice : Le Pleurer-Rire dont il fait lire les manuscrits à Sony Labou Tansi afin de recueillir son opinion. Or, ce dernier se trouve dans une intense réflexion au cours de laquelle, peu à peu, il prend conscience des limites de ses premiers romans, tous inédits. Hybrides, ils tiennent de l'autofiction, du pamphlet et de l'essai politique et ne correspondent plus au projet qu'il ambitionne de mener à bien : créer une œuvre si puissante qu'elle sera en mesure d'influencer les générations à venir. La rédaction du Mort te dit adieu toi qui restes vivant est une étape importante de son cheminement. Élaboré lors d'une crise morale toujours plus intense, son projet-monstre entremêle des bribes de sa nouvelle poétique romanesque et ses mythes personnels passés au filtre de la lecture du Pleurer-Rire.
Il ne reste aucune trace écrite, hélas, des échanges entre les deux écrivains, ni correspondances, ni manuscrits annotés, ni notes de lecture. Dans ces conditions, comment vérifier notre hypothèse - les rédactions du Mort te dit adieu toi qui restes vivant et de ses avatars se sont constituées à partir de l'appropriation d'un élément exogénétique, Le Pleurer-Rire? L'intrigue fournit un premier indice, bien maigre, il faut le reconnaître : il y est question des relations entre le personnage principal et son mentor, qui prend les traits d'Henri Lopes. Dans Le Mort te dit adieu toi qui restes vivant, Cataeno Pablo et son digne ami Henrico Vermos Diaz, ancien enseignant de l'École normale supérieure, ministre et écrivain ${ }^{15}$, sont tous deux victimes de la folie meurtrière d'un autocrate.

\section{IMEC, SEL 36456.}

15. On reconnaît, à grands traits, la trajectoire d'Henri Lopes. Après avoir enseigné l'histoire au sein de l'École normale supérieure d'Afrique centrale (ENSAC), il est nommé directeur général de l'enseignement avant de commencer une brillante carrière ministérielle. 
Comment «l'envie d'écrire comme Henri Lopes 16 » évoquée dans la préface du manuscrit transparaît-elle? Ce sont les difficultés de Sony Labou Tansi à élaborer une intrigue structurée qui permettent de formuler une réponse. Le cadre dans lequel s'inscrivent les mésaventures de Cataeno Pablo et d'Henrico Vermos Diaz achoppe sur un obstacle : à l'instar des romans précédents de Sony Labou Tansi - La Vie et demie est la seule exception à ce modèle -, l'intrigue peine à se stabiliser. Caché en raison des persécutions politiques dont il est l'objet, Cataeno Pablo est condamné à se remémorer le passé, notamment la geôle où il avait été un temps enfermé. Le récit s'enlise rapidement et, dans les premières pages, survient un commentaire largement métatextuel : «Suis pas un excellent narrateur ${ }^{17}$ ». Les échos du Pleurer-Rire interviennent précisément pour échapper au ressassement et au spectre du blocage. La lecture du manuscrit d'Henri Lopes est un aiguillon d'autant plus puissant qu'elle n'est assujetie à aucune note. Les débuts de Sony Labou Tansi, en effet, sont marqués par une représentation complexe de l'activité créatrice. Le besoin de tuteurs espérés, attendus, destinés à se transformer en conseillers littéraires, coexiste avec le rêve démiurgique de créer sans maître et de s'engendrer seul dans la matrice du verbe. En contrepoint de cette fiction de l'originalité, la composition de notes de lecture et de florilèges de citations placerait Sony Labou Tansi dans des positions qu'il exècre - celles de l'élève, du commentateur, voire du plagiaire.

Comment s'opère le rapport entre une exogenèse mentale et une dynamique endogénétique? Avant la phase de textualisation, tout se joue dans l'esprit de l'écrivain : intériorisés, les éléments intertextuels font l'objet de transformations via des processus de remémoration qui contribuent, dès ce stade, à les reconfigurer ${ }^{18}$. Lorsque vient le temps de la rédaction d'une nouvelle version, Sony Labou Tansi a déjà ingéré, en quelque sorte, les fragments exogénétiques qu'il a fait siens. À partir de Machin la Hernie, les changements structuraux paraissent inspirés du Pleurer-Rire et l'intrigue, pour reprendre un concept métatextuel qui scande sa correspondance, semble plus assise. Cataeno Pablo disparaît et laisse le premier plan au dictateur Martillimi Lopez, avatar délirant de l'un des personnages du Pleurer-Rire, Tonton Bwakamabé Na Sakkadé - c'est le monologue débondé de Lopez qui va porter cette nouvelle version. Les obsessions intimes de Cataeno Pablo sont escamotées au profit d'une intrigue fondée sur des échos avec Le Pleurer-Rire : la violence des autocrates, leurs relations troubles avec l'ancienne puissance coloniale, l'importance croissante de conseillers spéciaux français - Gourdain chez Henri Lopes, Vauban chez Sony Labou Tansi - et d'incessantes contestations populaires forment une trame commune.

Cette appropriation se laisse deviner dans la dédicace de L'État honteux, la contexture des intrigues depuis Le Mort te dit adieu toi qui restes vivant, et le jeu permanent avec l'identité d'Henri Lopes. D'Henrico Vermos Diaz à Martillimi Lopez en passant par la monstrueuse Hernie dont ce dernier est affublé, on ne peut s'empêcher d'entendre et de voir, directement ou en vertu d'un procédé anagrammatique, le nom et le prénom d'Henri Lopes sans cesse décomposés et recomposés. Modeler des éléments intertextuels selon des logiques essentiellement mentales et jouer avec l'identité de son ami constituent pour l'écrivain une manière de mettre fin à toute distorsion entre les représentations et les réalités de sa création. Il est bien Sony Labou Tansi l'indépendant, la forte tête, résolu à n'avoir aucun maître.

\section{Faux-frères, doubles et rivaux}

\section{Bantsimba, banzouzi}

La littérature imaginée collectivement a des conséquences sur les manières d'être et de se penser écrivain. Henri Lopes a beau refuser d'être qualifié de père malgré son rôle de modèle et l'autorité que lui ont conférée ses préfaces, une dynamique filiale, voire patrimoniale, a régulé les relations littéraires entre les écrivains et orienté leurs processus de création. Les couples formés par les aînés et leurs cadets ne sont toutefois pas les seuls à composer la

16. Sony Labou Tansi, Le Mort te dit adieu toi qui restes vivant, Bibliothèque francophone multimédia de Limoges, RES. PF SLT 38, p. 4. 17. Ibid., p. 18.

18. Dans un entretien avec le journaliste Apollinaire Singou-Basseha, Sony Labou Tansi décrit ce premier temps purement mental de la création qu'il appelle «le travail de l'écriture inconsciente». Nicolas MartinGranel, Greta Rodriguez-Antoniotti (dir.), «Le métier d'écrivain selon Sony Labou Tansi. Extraits des entretiens radiophoniques avec Apollinaire Singou-Basseha», Études littéraires africaines, op. cit., p. 32 (en ligne sur erudit.org). 
phratrie : une fiction fraternelle, parfois gémellaire, a donné lieu à deux types de créations exogénétiques. D'une part, une généalogie mouvante conduit les pères et les fils à devenir jumeaux, bantsimba et banzouzi en kikongo ${ }^{19}$, avec tous les miroitements que ce scénario identitaire et électif suppose. De jeux de miroirs en mises en abyme, des circulations intertextuelles trament une réflexion commune sur la figure du double. D'autre part, en vertu d'un relais établi du réel à l'imaginaire, les écrivains transforment leurs frères fantasmatiques en personnages dans des récits où intertextualité et métatextualité s'enchevêtrent. Tantôt patronymes et pseudonymes y sont à peine fictionnalisés - Martial 20, Bamba, Bemba Okaka, Bemba-Okakalou ou Marcello Sonitansi sont quelques-unes des identités égrenées -, tantôt aucun cryptage n'intervient à l'image d'une nouvelle de Maxime Ndébéka, «Le bon droit de la chèvre», où les grands noms de la phratrie sont convoqués.

Où chercher l'origine de ces constructions discursives? Au confluent de l'écriture et de la vie, de l'art et de l'amitié, dans une réalité tout à la fois littéraire, humaine et sociale, et désignée, selon le concept que Rémy Ponton a élaboré à la suite des travaux de Max Weber, par le terme de «communauté émotionnelle». Les préfaces, les dédicaces et les réseaux intertextuels s'inscrivent dans une économie symbolique du don et du contre-don, mais l'imaginaire de la fraternité a bien eu des effets sur les démarches créatrices. C'est parce que Sylvain Bemba a aimé Sony Labou Tansi et ses écrits qu'une complicité littéraire est née, moteur d'une dynamique gémellaire partagée. Les déclarations d'amour sont récurrentes dans la phratrie car on aime son double, son frère d'âme, ce beau miroir flatteur qui renvoie, diffracte et diffuse une image enchantée de soi. Bon camarade, Sylvain Bemba se fait d'ailleurs une spécialité de publier régulièrement, dans l'hebdomadaire La Semaine africaine, des articles élogieux sur les œuvres de ses pairs.

Parfois confondus en raison des sonorités voisines de leurs pseudonymes, Tchicaya U Tam'si et Sony Labou Tansi sont l'une des illustrations les plus abouties de ces affinités électives. En septembre 1977, à la lecture du recueil Le Pays intérieur que le jeune homme lui a fait parvenir dans un curieux colis - un paquet grossièrement emballé avec du fil électrique -, Tchicaya U Tam'si ressent une impression de familiarité et écrit avec émotion «je vous reconnais frère $^{21} \gg$. Quelques années plus tard, dans leurs hommages respectifs, «Portrait d'un écrivain par un autre», «Projet de préface à la mort d'un frère d'âme » et «Le pays intérieur du Prince Tchicaya U Tam'si », les deux écrivains se pensent au miroir de l'autre, au point de ne former qu'une entité. Jumeaux, assurément, ils le sont. Enfants géniaux, isolés et incompris, puis poètes maudits, victimes de leur «mauvais sang 22 », ils s'aiment, s'admirent, s'impressionnent le plus littéralement possible, et s'imaginent une filiation commune choisie hors de la phratrie - celle de Rimbaud.

\section{Dramaturgie de la généalogie}

En deçà du scénario de la petite société harmonieuse, il faut faire un sort aux malentendus et aux rivalités qui ont été l'une des facettes de la phratrie. Deux voies s'ouvrent aux chercheurs désireux d'analyser les logiques d'individualisation mises en œuvre pour se démarquer, se faire un nom et surpasser ses pairs. Une approche sociologique cernerait comment les réussites diverses au sein du «système littéraire francophone », selon la terminologie de Pierre Halen ${ }^{23}$, ont favorisé les mésententes : certains écrivains ont publié leurs œuvres dans de prestigieuses maisons d'édition parisiennes, quand d'autres, davantage légitimés sur un plan local, ont pu éprouver le sentiment de ne pas avoir été reconnus internationalement. Au cours de l'hiver 1978, Sony Labou Tansi

19. L'Autre monde. Écrits inédits, textes choisis et réunis par Nicolas Martin-Granel, Bruno Tilliette, Paris, Éditions Revue Noire, coll. «Soleil», 1997, p. 150.

20. Martial Malinda est l'un des nombreux pseudonymes de Sylvain Bemba

21. Lettre inédite inventoriée par Nicolas Martin-Granel et Julie Peghini au cours d'un séjour de recherche à Brazzaville, décembre 2017.

22. "Mauvais sang» est l'une des sections d'Une saison en enfer. L'expression inspire à Tchicaya U Tam'si le titre d'un recueil publié en 1955, Le Mauvais sang. Organique, la poétique de Sony Labou Tansi se fonde sur un imaginaire sanguin.

23. Pierre Halen, « Notes pour une topologie institutionnelle du système littéraire francophone», dans Littératures et sociétés africaines. Regards comparatistes et perspectives interculturelles. Mélanges offerts à János Riesz à l'occasion de son soixantième anniversaire, études réunies par P. S. Diop et H.-J. Lüsebrink, Tübingen, Gunter Narr Verlag, 2001, p. 55-68. Pierre Halen, «"Le système littéraire francophone" : quelques réflexions complémentaires", dans J.-M. Moura, L. d'Hulst (éd.), Les Études littéraires francophones : états des lieux, Villeneuve-d'Ascq, Éd. du Conseil scientifique de l'université Charles-de-Gaulle-Lille III, 2003, p. 25-38. 
et son conseiller littéraire au Seuil, Luc Estang, mettent la dernière main à la publication de La Vie et demie. L'écrivain, dans une lettre datée du 11 décembre, confie son embarras à son éditeur; Sylvain Bemba, qu'il n'ose décevoir, lui a proposé un projet de préface. Luc Estang le refuse et s'en explique en ces termes :

Je ne vous ai pas accusé réception de la préface de Monsieur Sylvain Bemba. Elle est excellente mais, à notre avis, superflue. Outre qu'il n'est pas d'usage de publier une préface à un roman, surtout quand le signataire pour le public français n'est pas plus connu que l'auteur, votre livre est capable de se défendre tout seul24.

Le coup est rude. Considéré au Congo comme un classique, lauréat de prix francophones et publié par des éditeurs spécialisés dans les littératures africaines ${ }^{25}$, Sylvain Bemba, aux yeux de l'institution littéraire française, n'existe pas. La dédicace de La Vie et demie est à reconsidérer. Reconnaissant envers Sylvain Bemba mais conscient de la politique du Seuil et des rapports de force à l'œuvre dans le «système littéraire francophone», Sony Labou Tansi a-t-il pensé sa dédicace comme le seul acte de piété filiale possible?

Une seconde approche, située dans le champ de la poétique, consisterait à mesurer les effets des conflits et des dissensions sur le plan créatif. La dynamique collaborative de la phratrie, qui s'est manifestée au travers de pratiques exogénétiques, a généré des tensions. La circulation d'éléments intertextuels a aidé des écrivains à relancer, voire amorcer, des processus rédactionnels, mais les réussites contrastées au sein de la phratrie et des querelles littéraires ont constitué l' exogenèse dialogique en espace conflictuel. À peu de chose près, c'est là l'intrigue du «Bon droit de la chèvre», nouvelle publiée par Maxime Ndébéka dans le recueil Vécus au miroir. Tsiak-Tsiang, son héros, est la malheureuse victime de contrefaçons systématiques. Avant même que ses écrits en gestation ne soient publiés, alors qu'ils sont encore des brouillons ouverts à toutes les possibilités, ses rivaux se les approprient par de mystérieux procédés et en tirent toute la gloire.

À la lumière de ce récit ironique, il ne s'agit pas de faire des écrivains de la phratrie des plagiaires impénitents, mais d'examiner comment, en adoptant une approche poétique et sociologique, des procès intertextuels ont été les catalyseurs d'une dramaturgie de la généalogie. D'un écrit à l'autre, des miroitements intertextuels ont favorisé des univers fondés sur un fantasme majeur : celui du plagiat 26 . L'absorption endogénétique est un vol d'autant plus insupportable que l'écrivain qui s'en rend coupable est, selon les configurations, un frère, un fils ou un père.

Le statut de Sony Labou Tansi, un temps fils chéri, indique comment des dynamiques poétiques et des impulsions fantasmatiques se sont imbriquées à l'échelle d'un groupe. Enfant possessif, ingrat et sournois dans certains écrits de Maxime Ndébéka ${ }^{27}$, anthropophage sous la plume de Sylvain Bemba 28 et cyclope aux yeux de Tchicaya U Tam'si ${ }^{29}$, il enjôlerait ses pères avant de les dévorer. Sa poétique du ventre, qui assimile, digère et transforme les écrits de ses aînés, suscite, peu à peu, un malaise. Lorsqu'Henri Lopes, à la fin des années soixante-dix, l'invite à lire le manuscrit du Pleurer-Rire, son souhait est de connaître l'opinion de ce bouillonnant cadet. Or, les rédactions du Mort te dit adieu toi qui restes vivant et de ses avatars soulignent une autre réalité collaborative, sans doute imprévisible pour Henri Lopes. La relation littéraire à laquelle il a un temps songé fait l'objet d'un gauchissement, voire d'une inversion, en vertu d'un jeu de miroirs qui, quelques années plus tard, va se répéter de manière amplifiée.

24. Lettre de Luc Estang à Sony Labou Tansi, 8 février 1979, IMEC, SEL 3645 6. Cette préface ne se trouve malheureusement pas dans les fonds de l'IMEC.

25. Les maisons d'édition qui ont publié les œuvres de Sylvain Bemba sont essentiellement l'ORTF-DAEC, CLÉ, P.J. Oswald et les Nouvelles éditions africaines.

26. Anthony Mangeon, «Henri Lopes : l'écrivain et ses doubles », Présence francophone, no 1 , «Scénographies romanesques africaines de la modernité», vol. 78, 2012, p. 36-54 (en ligne sur crossworks.holycross. edu). Anthony Mangeon, Crimes d'auteur : de l'influence, du plagiat et de l'assassinat en littérature, op. cit.

27. Maxime Ndébéka, Vécus au miroir : huit nouvelles, Paris, Publisud, coll. «Traverses des espaces francophones», 1991. Boniface MongoMboussa, Tchicaya U Tam'si, le viol de la lune : vie et ceuvre d'un maudit, La Roque-d'Anthéron, Vents d'ailleurs, 2014, p. 119.

28. Sylvain Bemba, «Sony Labou Tansi et moi», Équateur, no 1, 1986, p. 52.

29. Tchicaya U Tam'si, «Portrait d'un écrivain par un autre», art. cit., p. 9. Nicolas Martin-Granel, «Les vies ultérieures de Tchicaya U Tam'si. Post-scriptum à "Tchicaya Passion" ", Continents Manuscrits, no 4, «La matière Congo », P. Leroux, N. Martin-Granel, C. Riffard (dir.), 2015, en ligne sur openedition.org. 
Au début des années quatre-vingt, Sony Labou Tansi, sa troupe du Rocado Zulu Théâtre et Roger Chemain travaillent, sans en avertir Henri Lopes, à une réécriture de son recueil de nouvelles Tribaliques, lauréat, en 1972, du Grand Prix littéraire d'Afrique noire. Cette version dramaturgique, qui a pour titre Ils sont déjà là, est composée dans la perspective d'un prix diffusé sur Radio France Internationale : le Concours théâtral interafricain. Manuscrits, tapuscrits et d'éventuelles notes de lecture ont disparu, mais non toutes les archives relatives à cette entreprise. La pièce radiophonique numérisée par l'INA, des correspondances et des articles 30 suggèrent quelques pistes pour reconstituer la genèse et la réception d'une création qui, bien que collective, a eu essentiellement pour maître d'œuvre Sony Labou Tansi :

Un député-maire qui fait profession de féminisme est, dans la vie, un père tyrannique pour ses filles, un mari jaloux et un patron méprisant pour sa domestique. Par sa faute, l'enfant de cette dernière mourra, tandis que le fiancé de l'une de ses filles (qu'il repousse comme étant d'une autre ethnie) sera torturé à mort en prison... ce qui dérange passablement la belle cérémonie au cours de laquelle $\mathrm{M}$. le député doit être décoré de la médaille du féminisme ${ }^{31}$.

Dans cette brève présentation d'Ils sont déjà là, on reconnaît plusieurs nouvelles de Tribaliques : l'hypocrisie, la misogynie et la morgue du politicien Ngouakou-Ngouakou («Monsieur le Député»), la violence des rapports sociaux («L'avance»), les amours malheureuses de deux jeunes gens («Ah Apolline !») et la terreur instaurée par l'usage de la torture («Le complot»). Les quatre nouvelles d'Henri Lopes ont été dramatisées et condensées pour former une intrigue unique. Parfois intitulé Tribaliques, Ils sont déjà là est tout à la fois un hommage et une duplication : des nouvelles à la pièce de théâtre, les personnages et les intrigues sont quasi identiques et, lorsqu'on prend en compte les réalités institutionnelles, la gémellité est également troublante puisqu'à l'œuvre récompensée par le Grand Prix littéraire d'Afrique noire se superpose celle distinguée par le prix du théâtre vivant. L'absence des documents de travail interdit d'approfondir l'analyse, toutefois le seul résumé d'Ils sont déjà là donne de précieuses informations. La démarche ne correspond plus aux processus mémoriels utilisés pendant le continuum qui a mené du Mort te dit adieu toi qui restes vivant à L'État honteux; à la faveur du travail collectif, sans doute y a-t-il eu d'autres phases exogénétiques au cours desquelles le recueil Tribaliques a été annoté pour être ensuite démarqué avec une telle précision.

Les tapuscrits conservés par Henri Lopes à partir du milieu des années quatre-vingt-dix révèlent les conséquences de ces rivalités mimétiques sur son activité littéraire. Après son départ du Congo, comment a-t-il élaboré une poétique et une figure d'écrivain impossibles à dupliquer dans la collectivité de la phratrie? Parmi différents procédés, se distingue une pratique située aux confins de l'exogenèse : l'intratextualité. Au fil de ses romans, Henri Lopes ne se contente pas d'aménager échos et jeux de miroirs. Dans certains tapuscrits, les formes initiales des intrigues suggèrent la dimension structurante et puissamment réflexive du réemploi de son propre matériau. Organisée autour de voix narratives féminines et masculines, l'une des premières versions d'Une enfant de Poto-Poto, Blanc Manioc, débute par les considérations de Franceschini, personnage dont le métissage éveille autour de lui fascination, méfiance et soupçons. Les premières pages du tapuscrit dépeignent des souvenirs lointains : une jeunesse en partie passée chez «Tantine Ginette», une idylle naissante avec Aurore qui passe pour être une cousine, et l'émotion suscitée par un Paris métis sur lequel règne la chanteuse Kolélé, célèbre pour son spectacle à La Canne à sucre où la suit régulièrement son amant, un métis afro-asiatique appelé Victor-Augagneur Houang.

De mon passé, j'avais gommé ce que j'avais été et ne savais plus si je devais avoir honte ou revendiquer les années vécues dans le quartier de Bangassou. Pourtant, malgré cette insolence, j'aimais à me rendre chez Tantine Ginette et Tonton Gaston. Peut-être pour y retrouver l'ambiance d'une enfance dérobée. C'était aussi l'occasion d'y déguster la cuisine du pays aux sauces riches et pimentées. Et puis il y avait Aurore,

30. Il s'agit de la correspondance de Sony Labou Tansi avec l'universitaire Sonia Almeida, évoquée par Nicolas Martin-Granel dans «Saudades : le Brésil de Sony Labou Tansi», Études littéraires africaines, no 43, «Afrique-Brésil», D. Delas (dir.), 2017, p. 105-131, en ligne sur erudit. org. Arlette Chemain-Degrange, «Du livre à la scène : Les Tribaliques d'Henri Lopes par le théâtre congolais du Rocado », Recherche, pédagogie, culture, no 63, juillet-août-septembre 1983, p. 91-94. Gaspard Pan, «Habits neufs pour un spectacle», Mweti, no 895, 29 novembre 1983, p. 8-9.

31. La présentation de la pièce est accessible à l'Inathèque, dans les archives radiophoniques de Radio France Internationale. 
la fille de Tantine Ginette et de Tonton Gaston. Un type de métissage inattendu : une Dravidienne du continent noir! La peau sombre - au teint de sapotille disaient les Antillais - elle avait les cheveux soyeux qui descendaient jusqu'à la taille, à la manière des Océaniennes, des Indiennes et des filles de Madagascar. Quoique taquine et malicieuse, elle éveillait en moi des fantasmes. Mais je me réfrénais. «Aurore, c'est ta sœur, Momo!». M'amouracher d'elle eût été incestueux. En fait, Tantine Ginette n'était pas la sœur de ma mère. Aucun lien de parenté entre elles. Chez nous, les enfants appellent tonton et tantine les proches de la famille. Une convention qui les imprègne tellement qu'ils sont persuadés qu'il existe de véritables liens de sang avec la tribu des tontons et des tantines. Tous les enfants sont nos frères et sœurs.

Un monde bigarré se donnait rendez-vous chez Tonton Gaston et Tantine Ginette. Outre des métis - du pays et d'ailleurs - on y rencontrait des Africains et des Antillais. Quelques Blancs aussi dont la présence me gênait. Je craignais qu'ils ne me prissent pour un des leurs. À cause de la couleur de ma peau. C'est chez Tantine Ginette que j'ai fait la connaissance d'un ancien gouverneur des colonies, aujourd'hui ambassadeur de France en Afrique noire; de nombreux hommes d'affaires (français, portugais, libanais, africains); tout ce que Paris pouvait contenir de métis. J'ai oublié leurs noms, hormis celui de la fameuse chanteuse Kolélé, qui allait devenir le chef de protocole de Patrice Lumumba, et que j'allais écouter, à l'époque où elle se produisait à La Canne à sucre, une boîte de nuit black de Montparnasse. À la fin de son tour de chant, un métis afrasien, Victor-Augagneur Houang, avait le privilège d'être son cavalier pour les rumbas et les biguines 32 .

Ce passage de Blanc Manioc est entièrement fondé sur des éléments exogénétiques puisés par Henri Lopes dans ses romans précédents. On songe aux lieux - Vouragan, dans Le Chercheur d'Afriques, est originaire de Bangassou, et La Canne à sucre est l'établissement où se produit Kolélé dans Le Lys et le flamboyant -, aux personnages, avec la mention de Kolélé et de Victor-Augagneur Houang, et aux situations. Les aléas d'une «enfance dérobée», les rapports savoureux de jeunes gens avec leur parentèle, la construction de personnages métis qui s'interrogent sur leur identité plurielle et les réalités des sociétés coloniales et postcoloniales envisagées au prisme des relations franco-africaines constituent le matériau de ses romans précédents.

Dans la version finale intitulée Une enfant de Poto-Poto, ce passage disparaît de même que la narration se simplifie - Kimia conte seule son histoire et celle de Franceschini.
De Blanc Manioc à Une enfant de Poto-Poto, l'histoire d'une entrée en écriture se déploie : pour aiguiser son désir créatif et inventer des noyaux narratifs auxquels vont s'adjoindre descriptions, péripéties et dialogues, Henri Lopes recourt d'abord à des fragments de ses écrits précédents qu'il va peu à peu recomposer, parfois jusqu'à l'effacement. Selon la manière dont ils sont agencés, ces embrayeurs donneront naissance à des séquences elles aussi destinées à être explorées dans des chantiers futurs :

Cela dit, on peut effectivement noter que toutes mes histoires sont des variantes de la même histoire. Tout récemment, Sembène Ousmane aurait dit : «Chaque film d'un cinéaste est le brouillon du suivant. » Je crois que tout romancier peut souscrire à cette formule heureuse. [...] C'est une espèce de système comme jadis la publicité de Dubonnet présentait l'image d'un chat enroulant une bouteille de Dubonnet sur laquelle il y avait une autre image d'un chat enroulant une bouteille et ainsi de suite à l'infini; ou bien comme ces poupées russes, les matriochkas ${ }^{33}$.

Cette dynamique circulatoire n'est donc ni le fruit du hasard, ni le fait d'un romancier oublieux, mais la caractéristique majeure d'un univers qui s'amplifie par analogie et réactualisation de ses virtualités. L'élaboration d'un nouveau projet et sa textualisation ne semblent possibles qu'à partir de fragments exogénétiques destinés à introduire l'écrivain dans un paysage complexe, tout à la fois familier et étranger. La tension entre le même et l'autre, entre le passé et le présent, entre l'exogenèse et l'endogenèse, favorise la progression de chantiers créatifs portés par un rêve : une écriture dépourvue de bornes et de limites puisque personnages, intrigues et situations se prolongent indéfiniment, à l'image de Kolélé et de son amant qui obsèdent si bien leur créateur qu'ils opèrent un retour dans Blanc Manioc. Il ne faut pas sous-estimer les conséquences symboliques de cette démarche. Considérer ses œuvres publiées comme les brouillons de ses écrits à venir et les convertir en éléments exogénétiques voués à nourrir de nouveaux projets installent l'écrivain au centre de sa création et suppriment toute rivalité. Devenu son propre modèle, Henri Lopes contrôle, sur le plan fantasmatique, les contrefaçons et faux en écriture

32. Henri Lopes, Blanc Manioc, Fonds Henri Lopes, p. 14-15.

33. «Entretien de Boniface Mongo-Mboussa avec Henri Lopes », 31 octobre 1997, disponible sur Africultures.com. 
dont il pourrait être l'objet. Que signifie se réécrire ? Frayer des sentiers créatifs toujours à venir, conjurer une phratrie parfois dévoratrice et créer une fiction de la démiurgie - être tout à la fois son maître, son double et son épigone.

Le paradigme de l'enquête convient tout particulièrement à une étude génétique de la phratrie. L'incomplétude des archives impose de multiplier les pistes pour recomposer des étapes parfois manquantes, de proposer des hypothèses dont certaines demeurent difficilement vérifiables et d'analyser des traces textuelles parfois bien maigres. La question de l'exogenèse redouble les difficultés car les fonds documentaires conservent peu de traces de matériaux intertextuels et aucun élément de documentation ou d'observation. Quelques notes allographes sur un cahier, des dédicaces, des archives croisées avec des correspondances et des témoignages, des mises en abyme fictionnelles de l'activité exogénétique : ce sont là les preuves dont le critique, devenu apprenti limier, se saisit à mesure qu'il redéfinit sa méthodologie.

Ces réalités ne doivent toutefois pas nous induire en erreur : des dynamiques exogénétiques ont joué un rôle de premier plan dans les cercles de la phratrie. La comparaison des indices amassés suggère l'existence d'une exogenèse intériorisée qui, selon Sylvain Bemba, Sony Labou Tansi et Henri Lopes, s'est essentiellement jouée «dans la tête» des écrivains. Au sein de la République populaire, les menaces politiques incitent à se départir de documents compromettants - dans l'un des tapuscrits de son dernier roman Petit Mao, Henri Lopes se livre à cette réflexion métatextuelle : «Ne jamais prendre de notes [...] constitue le maître-mot des citoyens désireux de ne pas avoir à subir les interrogatoires de la Sécurité d'État ${ }^{34}$. » Les affres traversées par le Congo nourrissent pourtant très largement les écrits de la phratrie selon ce modèle : textes annotés, notes et relevés sont proscrits au profit d'une textualisation et d'une fictionnalisation programmées dès les premières phases rédactionnelles.

La circulation de manuscrits a, quant à elle, favorisé des pratiques qui ont pris la forme de conseils mis en œuvre ou non le temps de l'écriture venu et de processus intertextuels. Selon les projets, les jeux de miroirs empruntent différentes modalités : théorisée par Sony Labou Tansi, une «écriture inconsciente», manière de reconfiguration mentale d'éléments intertextuels, des réécritures organisées autour de modèles et, dans le cas d'Henri Lopes, le réemploi systématique d'écrits précédents au fil de nouveaux chantiers. La notion d'angoisse de l'influence est précieuse pour comprendre les logiques pyschologiques qui ont prévalu dans une communauté littéraire partagée entre hommages et rivalités, piété filiale et désir de s'affirmer. L'exogenèse présente une caractéristique majeure : collaborative, dialogique, elle enserre les écrits dans un réseau commun où les transformations de fragments exogénétiques ont pu alimenter une dramaturgie familiale.

La phratrie a-t-elle été une fiction? Sans doute, mais une fiction productive qui, au gré des échanges, des rencontres et des lectures, a diffusé une manière d'être écrivain et de faire de la littérature.

34. Henri Lopes, Petit Mao, Fonds Henri Lopes, p. 194. 
Chercheuse sous contrat postdoctoral (EUR Translitterae, Université PSL), CÉLINE GAHUNGU est l'auteure de l'essai Sony Labou Tansi. Naissance d'un écrivain (CNRS Éditions, coll. «Planète Libre Essais», 2019) et d'articles consacrés aux littératures africaines francophones.

cgahungu@hotmail.fr

\section{À l'épreuve des pères : la phratrie congolaise}

Au début des années soixante, le Congo traverse une période instable marquée par des procès et des assassinats politiques, des coups d'État et des tensions ethniques avivées. Ces difficultés n'empêchent pas certains Congolais de se vouloir écrivains; à partir du milieu des années soixante, ce désir prend, chez quelques-uns, une dimension collective. Henri Lopes, Jean-Baptiste Tati Loutard et Maxime Ndébéka se rencontrent et commentent leurs écrits pour l'essentiel inédits. Sous la poussée des événements politiques, ce premier cercle s'élargit et commence une mue dont l'un des acteurs principaux est Sylvain Bemba. Romanciers, poètes et dramaturges créent un territoire littéraire commun, où, à l'abri des clivages idéologiques, manuscrits et idées s'échangent librement : la phratrie. Dans ce laboratoire créatif caractérisé par des échanges permanents, quelles sont les formes et les spécificités de l'exogenèse?

In the beginning of the 1960s, the Congo went through an instable period marked by political trials and assassinations, coups d'États, and rekindled ethnical tensions. These difficulties did not prevent certain Congolese from wanting to be writers. Beginning in the 1960s, this desire took on, in the case of some writers, a collective dimension. Henri Lopes, Jean-Baptiste Tati Loutard, and Maxime Ndébéka met and discussed what they were working on. Spurred on by the political situation, this first circle grew, evolving into a new form in which Sylvain Bemba played an important role. Novelists, poets and playwrights created a collaborative literary space where, sheltered from ideological differences, manuscripts and ideas were exchanged freely: phratry. In this creative laboratory of long-standing exchanges, what were the forms and the specific instances of exogenesis?

Anfang der 1960er Jahre befand sich der Kongo in einer labilen Phase, die durch politische Prozesse und Attentate, Staatsstreiche und verschärfte ethnische Spannungen gekennzeichnet war. Diese Schwierigkeiten hinderten einige Kongolesen nicht daran, Schriftsteller werden zu wollen; ab Mitte der 1960er Jahre nahm dieser Wunsch für einige eine kollektive Dimension an. Henri Lopes, Jean-Baptiste Tati Loutard und Maxime Ndébéka trafen sich und kommentierten ihre im Wesentlichen unveröffentlichten Schriften. Unter dem Druck der politischen Ereignisse weitet sich dieser erste Kreis aus und beginnt eine Veränderung, bei der einer der Hauptakteure Sylvain Bemba ist. Romanschriftsteller, Dichter und Dramatiker schaffen ein gemeinsames literarisches Gefilde, auf dem, geschützt vor ideologischen Trennungen, Manuskripte und Ideen frei ausgetauscht werden: „die phratrie“. Was sind die Formen und Besonderheiten der Exogenese in diesem kreativen Laboratorium, das von einem ständigen Austausch geprägt ist?
A comienzos de los años sesenta, el Congo atraviesa un periodo inestable signado por procesos y asesinatos políticos, golpes de Estado y tensiones étnicas exacerbadas. Estas dificultades no impiden que algunos congoleses quieran ser escritores; a partir de mediados de los años sesenta, este deseo adquiere para algunos de ellos una dimensión colectiva. Henri Lopes, Jean-Baptiste Tati Loutard y Maxime Ndebeka se reúnen y comentan sus escritos, por lo general inéditos. Impulsado por algunos sucesos políticos, este primer círculo se agranda e inicia una transformación, de la cual Sylvain Bemba es uno de sus principales protagonistas. Novelistas, poetas y dramaturgos crean un territorio literario común, en el cual, al margen de los enfrentamientos ideológicos, intercambian libremente manuscritos e ideas: la fratria. En este laboratorio creativo caracterizado por los intercambios permanentes, ¿cuáles son las formas y las especificidades de la exogénesis?

No início da década de 60, o Congo atravessa um período instável marcado por julgamentos e assassinatos políticos, golpes de Estado e tensões étnicas exacerbadas. Estas dificuldades não impedem que alguns congoleses queiram ser escritores; a partir de meados dos anos 60, este desejo assume, para alguns, uma dimensão coletiva. Henri Lopes, Jean-Baptiste Tati Loutard e Maxime Ndébéka encontram-se e comentam os seus escritos na maioria inéditos. Sob pressão de acontecimentos políticos, este primeiro círculo alarga-se e dá início a uma mudança em que um dos atores principais é Sylvain Bemba. Romancistas, poetas e dramaturgos criam um território literário comum, onde, ao abrigo de divisões ideológicas, manuscritos e ideias são livremente trocados: a fratria. Neste laboratório criativo caracterizado por trocas permanentes, quais são as formas e especificidades da exogénese?

All'inizio degli anni sessanta, il Congo attraversa un periodo d'instabilità segnato da processi e omicidi politici, colpi di stato e vive tensioni etniche. Queste difficoltà non impediscono ad alcuni congolesi di immaginarsi scrittori e dalla metà degli anni sessanta questo desiderio prende una dimensione collettiva. Henri Lopes, Jean-Baptiste Tati Loutard e Maxime Ndébéka s'incontrano e commentano i loro testi, per la maggior parte inediti. Sotto la spinta degli eventi politici, questo primo cerchio si allarga e inizia una metamorfosi, di cui uno degli attori principali è Sylvain Bemba. Romanzieri, poeti e drammaturghi creano un territorio letterario comune, dove, al riparo delle differenze ideologiche, manoscritti e idee si scambiano liberamente: la fratria. In questo laboratorio creativo caratterizzato da scambi permanenti, quali sono le forme e le particolarità dell'esogenesi? 